\title{
ON THE RISK FACTORS OF THE YIELD IN THE CRYPTOCURRENCIES MARKET
}

\author{
Aleš Kozubík ${ }^{198}$ \\ https://doi.org/10.31410/itema.2018.507
}

\begin{abstract}
A cryptocurrencies are considered as digital assets that use strong cryptography to secure financial transactions, control the creation of additional units, and verify the transfer of assets. Bitcoin was released as the first and the most popular cryptocurrency in 2009. Since its release, over 4000 alternative cryptocurrencies have been released. After a period of gradual growth of the Bitcoin value, we were able to observe a dramatically increasing in its value at the beginning of this year. This was followed by a subsequent rapid decline to the value it keeps to this day. A similar development we can, however, observe with other cryptocurrencies. This paper presents the results of an empirical analysis of the nature of the factors that explain changes in yields of the ten most traded cryptocurrencies. Consistent with the analysis of the current currencies yield curves, the principal component method has shown that two or three factors are sufficient to explain most of the yield variation. These results are essential for hedging purposes.
\end{abstract}

Keywords: cryptocurrency, risk factors, yield volatility, principal components

\section{INTRODUCTION}

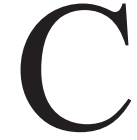

ryptocurrencies have become a full-fledged investment tool in a short period of their existence. Demand for these assets is still growing. In particular, the transition of 2017 and 2018 was characterized by a great interest in buying and the sharp rise in cryptocurrencies prices. However, it was also followed by a sharp decline. These turbulences in the cryptocurrencies market show that it is necessary to analyze their yields and the risks associated with them similarly as we do with other investments.

A cryptocurrency is in general considered as a digital asset designed to work as a medium of exchange that uses strong cryptography to secure financial transactions, control the creation of additional units, and verify the transfer of assets (see [2]). The formal definition of the cryptocurrency was given in [5]. According to Lansky, a cryptocurrency is a system that meets six conditions:

1. The system does not require a central authority; its state is maintained through distributed consensus.

2. The system keeps an overview of cryptocurrency units and their ownership.

3. The system defines whether new cryptocurrency units can be created. If new cryptocurrency units can be created, the system defines the circumstances of their origin and how to determine the ownership of these new units.

4. Ownership of cryptocurrency units can be proved exclusively cryptographically.

\footnotetext{
${ }^{198}$ University of Žilina - Faculty of Management Science and Informatics - Department of the Mathematical Methods and Operations Research, Univerzitná 8215/1, 01026 Žilina, Slovak Republic
} 
5. The system allows transactions to be performed in which ownership of the cryptographic units is changed. A transaction statement can only be issued by an entity proving the current ownership of these units.

6. If two different instructions for changing the ownership of the same cryptographic units are simultaneously entered, the system performs at most one of them.

Study of yield curve behavior has been an import part of financial market research as it provides us important information about the future expectation of growth, inflation, recession, etc. Modeling interest rates for the purposes of pricing of interest rate dependent cash flows and hedging the interest rate risk have been a topic of much interest to actuaries over recent decades (see for example [8], [4], [7] and [1]). These studies demonstrate both similarities and differences in international bond markets.

The aim of this study is to apply the factor analysis to determine the number of factors that explains the volatility of the yields in the cryptocurrencies market. Unlike conventional currencies, there does not exist the bond market for different maturities and the corresponding yield curves. Therefore, our work is based only on exchange rate gains and losses against the US dollar.

\section{DATA AND METHODS}

The data necessary for purposes of our analysis, we obtained from the CoinMarketCap portal [9]. This source provides data on exchange rates for a total of 2100 cryptocurrencies, with USD as the counter currency. This data is provided on a daily basis starting on April 28, 2013 , or later on the day on which the relevant coin was commenced. For purposes of our analysis, we have selected ten cryptocurrencies according to the highest market capitalization. Specifically, these were the following cryptocurrencies: Bitcoin, Ethereum, EOS, Litecoin, Cardano, Dash, Dogecoin, Monero, Nem, and Neo. The data was downloaded on October 6, 2018. So we have got datasets in length from 371 values for the shortest record of Cardano exchange rates to the longest series of 1986 rates for Bitcoin or Litecoin.

Figure 1 shows the exchange rate development of three cryptocurrencies, Bitcoin, Ethereum, and Monero. It is easily visible that the exchange rate development for all three selected currencies was very similar, with a peak on the break of the year 2018. A similar result we could observe also in the case of other relevant cryptocurrencies. It is, therefore, reasonable to expect that the returns on investments in individual cryptocurrencies are fundamentally influenced by common factors.

Let us assign the USD exchange rate of the cryptocurrency at time $t$ as $P_{t}$. The corresponding yield $y_{t}$ of the investment in the period from $t$ to $t+1$ we compute by formula:

$$
y_{t}=\frac{P_{t+1}-P_{t}}{P_{t}}
$$

In order to determine the factors that explain the substantial part of the yield volatility we apply the multivariate factor analysis (see for example [6]). That means, we will estimate the changes in the cryptocurrency yields in the form:

$$
\Delta y_{c t}=\sum_{i=1}^{n} \alpha_{c i} F_{i t}+\varepsilon_{c t}
$$

where: 
$\Delta y_{c t}$ is the change in the yield of the investment in cryptocurrency $c$ at time $t$, $F_{i t}$ is the value of the $\mathrm{i}$-th independent random factor at time $t$, $\alpha_{c i}$ is the factor loading for the $i$-th factor for cryptocurrency $c$ $\varepsilon_{c t}$ is the error term, representing the volatility unexplained by the factors.

Figure 1: Market values of three selected cryptocurrencies, expressed as their USD exchange rates. The observations are numbered from 0 on 2013 April, the 28-th, to 1986 on 2018 October, the 6-th. (Source: Own elaboration of data by [9].)

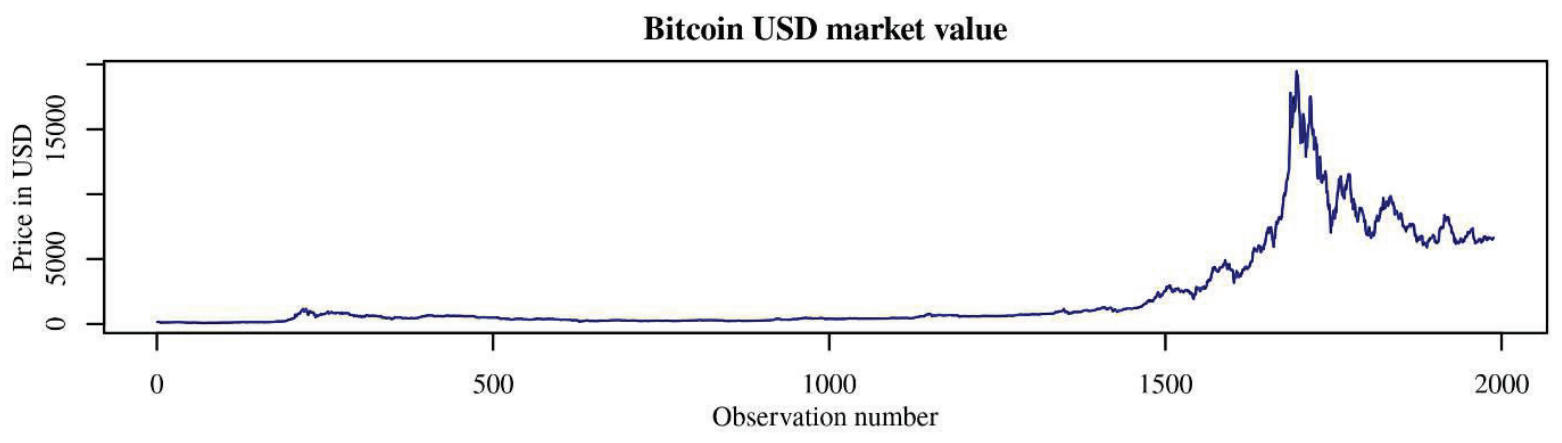

Ethereum USD market value

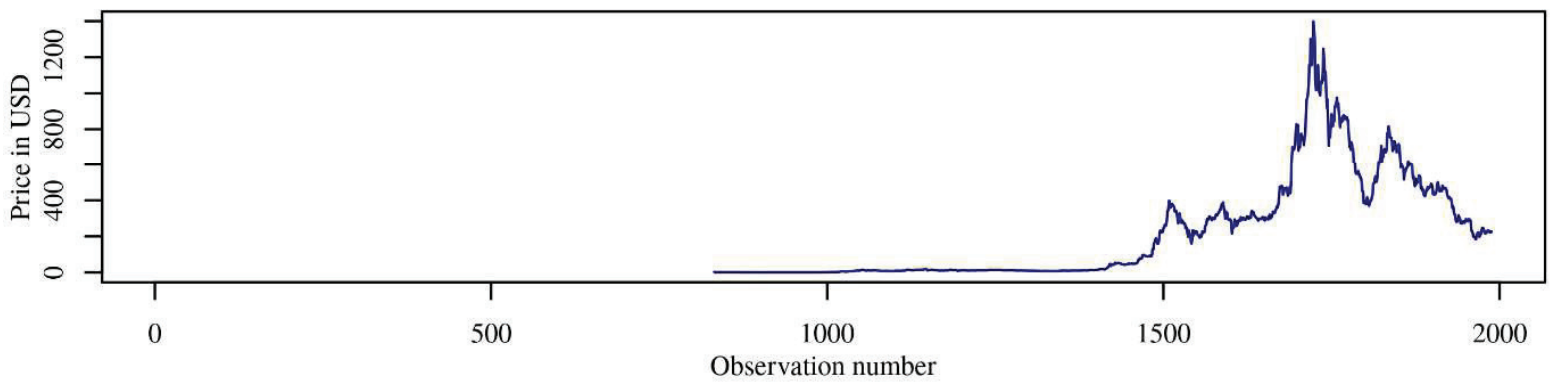

Monero USD market value

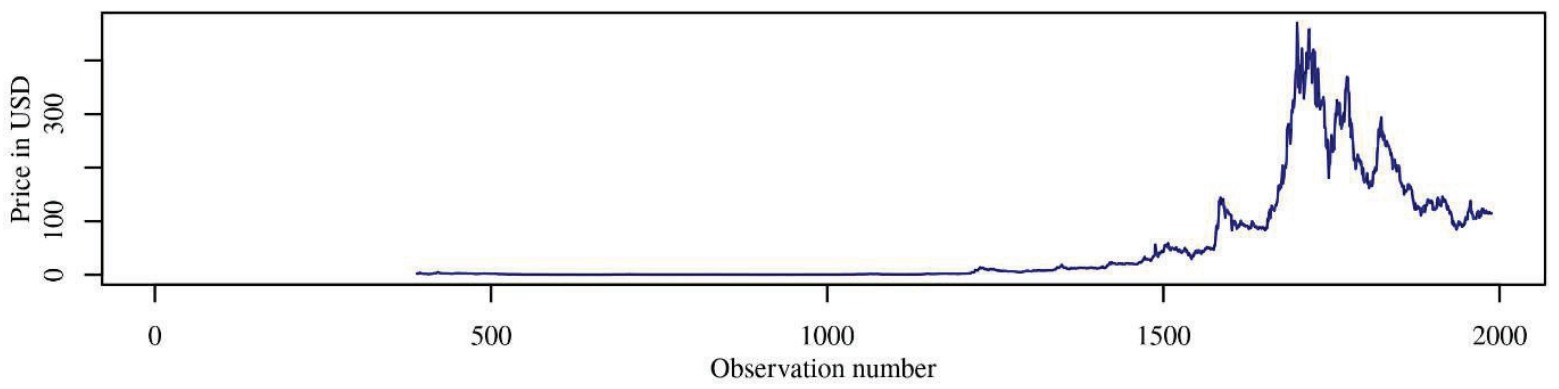

We estimated the factor loadings using the correlation matrix and principal component factor analysis. This method works also with the eigenvalues and eigenvectors of the correlation matrix. The most common steps can be described as follows:

1. Preparing the data, especially compute the correlation matrix.

2. Decide how many components to extract.

3. Extract and rotate the components.

4. Compute the factor loadings, communalities and portion of volatility that is explained by the factors.

5. Interpret the results.

Important role in the principal components factor analysis plays stating the number of the factors. Here are three the most frequently used methods: 
- The Kaiser-Harris criterion. It suggests retaining the components with eigenvalues greater than 1.

- Cattel scree test. The eigenvalues are plotted against their component numbers. Such plots typically demonstrate a bend or elbow, and the components above this sharp break are taken in account.

- Parallel analysis. We run simulations, extracting eigenvalues from random data matrices of the same size as the original matrix. If an eigenvalue based on real data is larger than the average corresponding eigenvalues from a set of random data matrices, that component is retained. (For more details see [3]).

\section{RESULTS}

The first step in the analysis is to compute the yields and simultaneously the changes in the yields for all cryptocurrencies. The essential sample characteristics for both are presented in Table 1. Look on the Table 1 confirms that all cryptocurrencies keep relatively stable value. The median values show, that there is a fifty-fifty chance in growth or decline and the positive values of the average daily yields are caused by short periods of dramatically growth that correspond to the Max columns in the Table 1.

Table 1: Selected sample characteristics of the yields and changes in the yields of the investments in the cryptocurrencies.

\begin{tabular}{l|r|r|r|r|r|r|r|r}
\hline \multirow{2}{*}{ Currency } & \multicolumn{4}{|c|}{ Yield } & \multicolumn{4}{c}{ Change in the yield } \\
\cline { 2 - 9 } & Mean & Median & St.dev. & Max & Mean & Median & St.dev. & Max \\
\hline Bitcoin & 0.002 & 0.003 & 0.044 & 0.435 & 0 & 0 & 0.062 & 0.572 \\
Ethereum & 0.007 & 0 & 0.074 & 0.497 & 0 & 0 & 0.101 & 0.758 \\
EOS & 0.010 & -0.003 & 0.126 & 1.730 & 0 & 0 & 0.166 & 1.762 \\
Litecoin & 0.004 & 0 & 0.075 & .281 & 0 & 0 & 0.103 & 1.327 \\
Cardano & 0.001 & -0.005 & 0.124 & 1.335 & 0 & -0.002 & 0.166 & 0.953 \\
Dash & 0.007 & -0.003 & 0.094 & 1.906 & 0 & -0.001 & 0.136 & 1.660 \\
Dogecoin & 0.005 & -0.003 & 0.095 & 1.894 & 0 & 0 & 0.126 & 1.414 \\
Monero & 0.005 & -0.001 & 0.0795 & 0.764 & 0 & 0 & 0.126 & 1.414 \\
Nem & 0.001 & 0 & 0.107 & 1.911 & 0 & 0 & 0.111 & 0.646 \\
Neo & 0.013 & -0.0046 & 0.139 & 1.022 & 0 & 0.003 & 0.156 & 2.045 \\
\hline
\end{tabular}

(Source: Own elaboration, data by [9].)

For the deeper analysis of the common factors influencing the yields it is necessary to compute the correlation matrix of the yield changes. In order to save some space, it is presented on Figure 2 , in the form of the heatmap with values. The values of the correlation coefficients are colored so that darker color represents the higher absolute value of the correlation. In order to determine the number of components retained, we draw a scree plot. The result is illustrated on Figure 3. We easily see, that all three methods state only two principal components to be sufficient to explain the variability of the yields. Really, we have only two eigenvalues $\lambda_{I}=5.524$ and $\lambda_{2}=1.030$, which are greater than 1 . On Figure 3 is also visible the sharp break at the second eigenvalue. Because the third eigenvalue is close to one $\left(\lambda_{3}=0.906\right)$, we include three factors in our calculations. These three principal factors then explain about $77 \%$ of the yields volatility. 
All numerical calculations have been made using the $\mathrm{R}$ statistical programming environment with the psych package. So we obtained the results that are summarized in Table 2. The corresponding root mean square residuals value (RMSR) of the model is 0.07 .

Figure 2: The correlation matrix of the yield changes for the investments in the cryptocurrencies.

\begin{tabular}{|c|c|c|c|c|c|c|c|c|c|c|}
\hline & 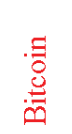 & 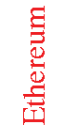 & 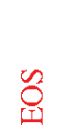 & 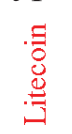 & 槖 & $\begin{array}{l}\frac{5}{\tilde{D}} \\
\stackrel{5}{0}\end{array}$ & $\begin{array}{l}\text { : } \\
8 \\
0 \\
8 \\
8 \\
0\end{array}$ & 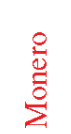 & 寻 & $\stackrel{8}{Z}$ \\
\hline Bitcoin & 1 & 0.65 & 0.43 & 0.63 & 0.36 & 0.52 & 0.6 & 0.6 & 0.58 & 0.19 \\
\hline Ethereum & 0.65 & 1 & 0.6 & 0.75 & 0.46 & 0.63 & 0.54 & 0.54 & 0.69 & 0.4 \\
\hline EOS & 0.43 & 0.6 & 1 & 0.5 & 0.32 & 0.41 & 0.37 & 0.37 & 0.49 & 0.35 \\
\hline Litecoin & 0.63 & 0.75 & 0.5 & 1 & 0.36 & 0.56 & 0.52 & 0.52 & 0.6 & 0.43 \\
\hline Cardano & 0.36 & 0.46 & 0.32 & 0.36 & 1 & 0.33 & 0.49 & 0.49 & 0.52 & 0.36 \\
\hline Dash & 0.52 & 0.63 & 0.41 & 0.56 & 0.33 & 1 & 0.49 & 0.49 & 0.68 & 0.28 \\
\hline Dogecoin & 0.6 & 0.54 & 0.37 & 0.52 & 0.49 & 0.49 & 1 & 1 & 0.54 & 0.34 \\
\hline Monero & 0.6 & 0.54 & 0.37 & 0.52 & 0.49 & 0.49 & 1 & 1 & 0.54 & 0.34 \\
\hline Nem & 0.58 & 0.69 & 0.49 & 0.6 & 0.52 & 0.68 & 0.54 & 0.54 & 1 & 0.34 \\
\hline $\mathrm{Neo}$ & 0.19 & 0.4 & 0.35 & 0.43 & 0.36 & 0.28 & 0.34 & 0.34 & 0.34 & 1 \\
\hline
\end{tabular}

(Source: Own elaboration.)

Figure 3: The scree plot for stating the number of the principal components. (Source: Own elaboration.)

Parallel Analysis Scree Plots

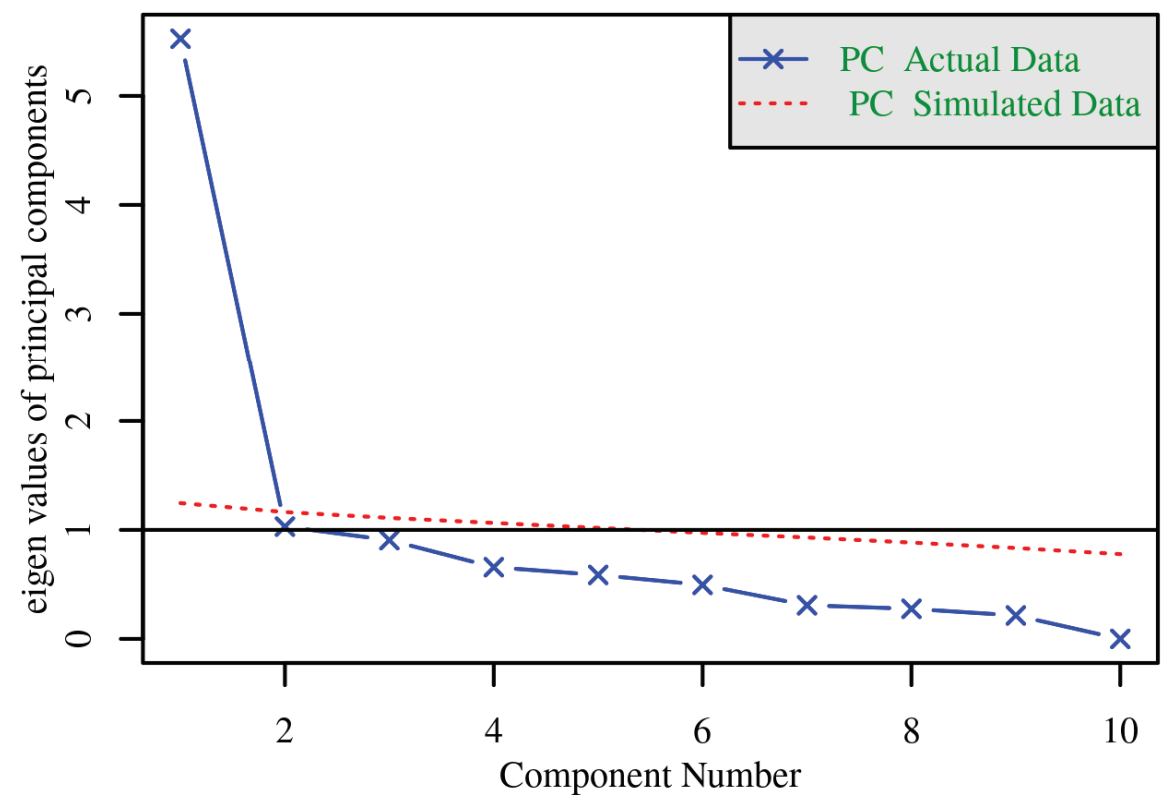


These three factors can be interpreted as explaining different types of change in the yields. The first factor affects the changes in the yield of all currencies in the same direction, so we can interpret it as a parallel shift factor.

Table 2: The factor loadings obtained by the principal component method with three explaining factors.

\begin{tabular}{|l|c|c|c|c|}
\hline Currency & Factor 1 & Factor 2 & Factor 3 & Uniqueness \\
\hline Bitcoin & 0.69 & 0.49 & -0.10 & 0.28 \\
\hline Ethereum & 0.82 & 0.27 & 0.26 & 0.19 \\
\hline EOS & 0.68 & 0.03 & 0.36 & 0.42 \\
\hline Litecoin & 0.76 & 0.26 & 0.25 & 0.29 \\
\hline Cardano & 0.21 & 0.50 & 0.52 & 0.43 \\
\hline Dash & 0.75 & 0.27 & 0.05 & 0.36 \\
\hline Dogecoin & 0.31 & 0.91 & 0.16 & 0.05 \\
\hline Monero & 0.31 & 0.91 & 0.16 & 0.05 \\
\hline Nem & 0.72 & 0.35 & 0.21 & 0.31 \\
\hline Neo & 0.20 & 0.12 & 0.88 & 0.17 \\
\hline
\end{tabular}

(Source: own calculations.)

The second factor has as well positive effect on all currencies, but here are greater differences as in the case of the first factor. Moreover, for some currencies it is very close to zero. So we can interpret this factor as the "curvature" factor.

The third factor has an opposite effect on Bitcoin and on the other currencies. So it can be interpreted as a "slope" factor since it changes the slope of the rates of return.

Compared with the studies of the yield curves for arbitrary currencies, the slope and curvature factors appear to be more important in explaining the volatility of the yields changes on the cryptocurrencies market. We can expect a single factor model to replicate only around $56 \%$ of the yields changes.

\section{CONCLUSIONS}

In this paper, we examine the common factor structure of the cryptocurrencies exchange rates against the USD returns corresponding to ten cryptocurrencies. We have applied the principal components analysis to identify the factors which are responsible for volatility of the yield curve. The study finds that the three principal component explains a major part of the total variance of exchange rates yields changes. Compared with studies for conventional studies three factors are sufficient to explain substantial part of the variance in the yields. It means, that a single factor mode, frequently used for the pricing purposes of the bonds denominated in the conventional currencies, is in this case insufficient nor for purposing of pricing, nor for hedging. For this reason it is considered essential that multi-factor models be used for immunization. 


\section{AKCNOWLEDGEMENT}

This work has been supported by the grant of the Slovak Educational and Cultural Agency KEGA 041ŽU-4/2017 "Experimental mathematics - accessible for all".

\section{REFERENCES}

[1] Ceballos, L. (2014). The Yield Curve Factors and Economic Surprises in the Chilean Bond Market. Revista de Análisis Económico, Vol. 29, No 2, 3-23.

[2] Chohan, U. W. (2017). Cryptocurrencies: A Brief Thematic Review, Discussion Paper, University of New South Wales, Canberra, August 4. Available at SSRN: https://ssrn.com/abstract=3024330 or http://dx.doi.org/10.2139/ssrn.3024330

[3] Hayton, J. C., Allen, D. G., Scarpello, V. (2004). Factor Retention Decisions in Exploratory Factor Analysis: a Tutorial on Parallel Analysis. Organizational Research Methods, 7(2), pp. 191-205. https://doi.org/10.1177/1094428104263675

[4] Juneja, J. (2012). Common factors, principal components analysis, and the term structure of interest rates. International Review of Financial Analysis 24, 48-56.

[5] Lansky, J. (2018). Possible State Approaches to Cryptocurrencies. Journal of Systems Integration, 9/1, pp.19-31.

[6] Reyment, R., A., Jöreskog, K., G. (1996). Applied Factor Analysis in the Natural Sciences. Cambridge: Cambridge University Press.

[7] Scherer, K., P., Avellaneda, M. (2002). All for one...one for all? A principal component analysis of the Latin American Brady bond debt from 1994 to 2000. Journal of International Theoretical and Applied Finance, 5, 79-107.

[8] Soto, G., M. (2004). Using Principal Component Analysis to Explain Term Structure Movements: Performance and Stability. In F. Tavidze, (Ed.), Progress in Economics Research 8. New York, NY: Nova Science Publishers, 203-226.

[9] https://coinmarketcap.com 